\title{
Passively Q-Switched Pulses Generation from Erbium-Doped Fiber Laser Using Lutetium Oxide as Saturable Absorber
}

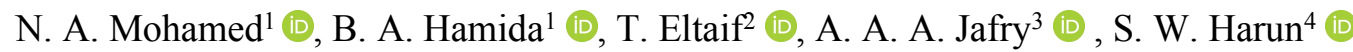 \\ ${ }^{1}$ Department of Electrical Engineering, Faculty of Engineering, International Islamic University Malaysia \\ (IIUM), Jalan Gombak, 53100 Kuala Lumpur, Malaysia, Eng.nasser.mohamed95@gmail.com, \\ belal@iium.edu.my \\ ${ }^{2}$ Department of Electrical and Electronics Engineering, Xiamen University Malaysia, Jalan Sunsuria, Bandar \\ Sunsuria 43900 Sepang, Selangor, Malaysia, tefosat@ieee.org \\ ${ }^{3}$ Department of Physics, Faculty of Science, Universiti Teknologi Malaysia, 81310 Skudai, Johor, Malaysia, \\ afiqarif@graduate.utm.my \\ ${ }^{4}$ Department of Electrical Engineering, Faculty of Engineering, University of Malaya, 50603 Kuala Lumpur, \\ Malaysia, swharun@um.edu.my
}

\begin{abstract}
In this paper, a configuration of a passive Q-switched Erbium-doped fiber laser (EDFL) using a Lutetium Oxide $\left(\mathrm{Lu}_{2} \mathrm{O}_{3}\right)$ thin film as a saturable absorber (SA) is experimentally implemented to generate high pulse energy with a high signal to noise ratio. A stable Q-switched pulse train is initiated at the input pump power of $30.442 \mathrm{~mW}$, and a maximum pulse energy of $16.11 \mathrm{~nJ}$ is obtained at an output power of $0.97 \mathrm{~mW}$, which confirms the stability of the pulses. It was possible to increase the repetition rate of the Q-switched laser from 31.25 to $60.2 \mathrm{kHz}$ as the pump power was raised from $30.442 \mathrm{~mW}$ to $71.652 \mathrm{~mW}$. Moreover, the pulse width decreased from $11.4 \mu$ s to $4.27 \mu$ s and $66.4 \mathrm{~dB}$ of the received signal-to-noise ratio at the radio frequency spectrum was achieved.
\end{abstract}

Index Terms - Q-switched, erbium-doped fiber laser, Lutetium oxide, fiber laser, saturable absorber.

\section{INTRODUCTION}

Fiber laser applications have opened numerous new implementations, and it has become easier and more compatible to use and design the laser. The greater fiber surface to volume ratio permits high heat dissipation performance [1]. Since the doped fiber has a longer period of usability compared to a standard laser resonator, it is possible to achieve a greater gain and thus a greater power output [2]. Because of the high output power and flexibility in usage and design, practical applications requiring Erbium-doped fiber (EDF) as a gain medium in the cavity have increased substantially in recent years. For instance, due to its capabilities in fiber sensor applications and optical communication, many scholars use EDF as their gain medium [3] - [4]. Also, dense wavelength division multiplexing (DWDM) systems utilize highest data rate in amplifying data channels [4]. In recent years, researchers have gained interest in Q-Switched fiber lasers acquired using docile techniques, because they are easy to generate and more solid in setup. The passive techniques are flexible and can trigger 
pulses without the need of an electronic controller [5]. Carbon nanotubes (CNTs) [6], black phosphorus (BP) [7], Semiconductor saturable absorbers (SESAMs) [8] and graphene [9] have been effectively utilized as intracavity-loss modulators for the passive production of pulsed lasers. After its first production in 1992, SESAMs was recorded to be the most outstanding SA for the following years. Nevertheless, they have noticeable defects like a high production cost, markedly bulky size, and narrow absorption bandwidth) [8]. It is worth to mention that, the major defect of the CNT SAs is the fact that the diameter largely affects its ability to absorb alongside its bandwidth. Meanwhile low optical absorption per layer is the major drawback in graphene, resulting a limitation to use it. On the other hand, BP depends on polarization and is a hydrophilic material that can interact with water easily. Therefore, establishing a BP SA is not easy because it requires complicated preparation and cautious handling [7].

Erbium as Rare Earth (RE) material for optical fibers is used to be doped as the cavity gain medium for producing a laser pulse. The method used to enhance the function of rare earth materials is to reap the benefits of the wave guiding medium path. An exceptional chemical compound of Lutetium (Lu) is the rare earth Lutetium oxide $\left(\mathrm{Lu}_{2} \mathrm{O}_{3}\right)$ which is one of the 17 chemical elements that are classified as Rare earth elements (REEs). Due to their several applications in various fields, these luminescent elements have attracted a lot of attention. They can be used in lamps and display devices, X-ray medical radiography, high-power artificial lights and solid-state lasers [10]. Moreover, REEs play an important role in the functionality of different modern commercial technologies, for example, electric vehicles (magnets and batteries), catalytic converters, wind turbines (magnets), fluorescent lighting (phosphors), and defense applications [11]. REEs have attracted interest from multiple nations since these chemical elements are critical and fundamental towards the improvement of modern green energy technologies as well as the economic well-being [11] - [12]. Despite the fact that REEs are abundantly available resources, they are generally widely dispersed and contained in small quantities, which contribute to environmental exhausting mineral extraction and energy intensive fine tuning processes and extraction. REEs have a magnetic and luminescent nature, which makes them desirable and often used at the industrial level. However, due to their relatively low concentrations in the environment, when highly concentrated materials are mined, REEs are co-products of this process. Furthermore, REEs usually are known for their high intensity and expensive prices as resources in their recovery process, when in comparison with other typical ores like coal or iron [11]. Among the rare-earth (RE) materials, there is a group of compounds called Sesquioxides, which are basically defined as oxides containing three atoms of Oxygen with two atoms of another element. These compounds are well known for being host materials because of their sufficient thermal conductivity and good chemical stability.

In order to detect various ionizing radiation, such as X-rays and gamma particles, Lutetium oxide is widely recognized as an effective host [10]. The high density of $\mathrm{Lu}_{2} \mathrm{O}_{3}\left(9.42 \mathrm{~g} / \mathrm{cm}^{3}\right)$ is the main reason of the high functionality of these detectors, which alongside the large atomic number of lutetium (71), 
grants a sufficient terminating energy for ionizing radiation [10]. Additionally, the cubic crystal structure of $\mathrm{Lu}_{2} \mathrm{O}_{3}$ helps to make the production of polycrystalline transparent ceramics attainable. In fact, this is largely significant, since the $\mathrm{Lu}_{2} \mathrm{O}_{3}$ high melting point of (approximately $2490^{\circ} \mathrm{C}$ ), makes the growth of a single crystal complex possible with normal growth methods [11], [12]. This article focuses on generating a Q-switched pulse laser in an Erbium-doped fiber laser cavity using $\mathrm{Lu}_{2} \mathrm{O}_{3}$ film as a saturable absorber with a coupling ratio of 10:90 to generate a high repetition rate, high pulse energy, high SNR, and short pulse width. The designed experimental procedure required no polarization controller, this technique offers a stable pulse generation without mechanical and environmental perturbations [13].

\section{PREPARATION AND NONLINEAR TRANSMISSION PROFILE OF SA MATERIAL}

The $\mathrm{Lu}_{2} \mathrm{O}_{3}$ powder was procured from Shanghai Xinglu Chemical Technology Co., Ltd. The powder owns a purity of $99.99 \%$, particle size of $50 \mathrm{~nm}$, density of $9.42 \mathrm{~g} / \mathrm{cm}^{3}$, and molecular weight of $397.94 \mathrm{~g} / \mathrm{mol}$. The synthesizing process starts by mixing $5 \mathrm{mg} \mathrm{Lu}_{2} \mathrm{O}_{3}$ powder with $50 \mathrm{ml}$ isopropyl alcohol. The stirring process took $24 \mathrm{~h}$ with $300 \mathrm{rpm}$ stirring speed at room temperature. The solution was then mixed with $120 \mathrm{ml}$ polyvinyl alcohol (PVA) solution, this process took place at ambient temperature. Finally, the prepared mixture was placed in a petri dish and left to dry for $48 \mathrm{~h}$. The nonlinear transmission profile of lutetium oxide film was captured by using a balanced-twin detector technique. Fig. 1 shows the developed SA owns a modulation depth of $4 \%$, a nonlinear loss of $3 \%$, and a saturable intensity of $32.03 \mathrm{MW} / \mathrm{cm}^{2}$. This indicates its ability to convert a continuous-wave laser into a Q-switching regime.

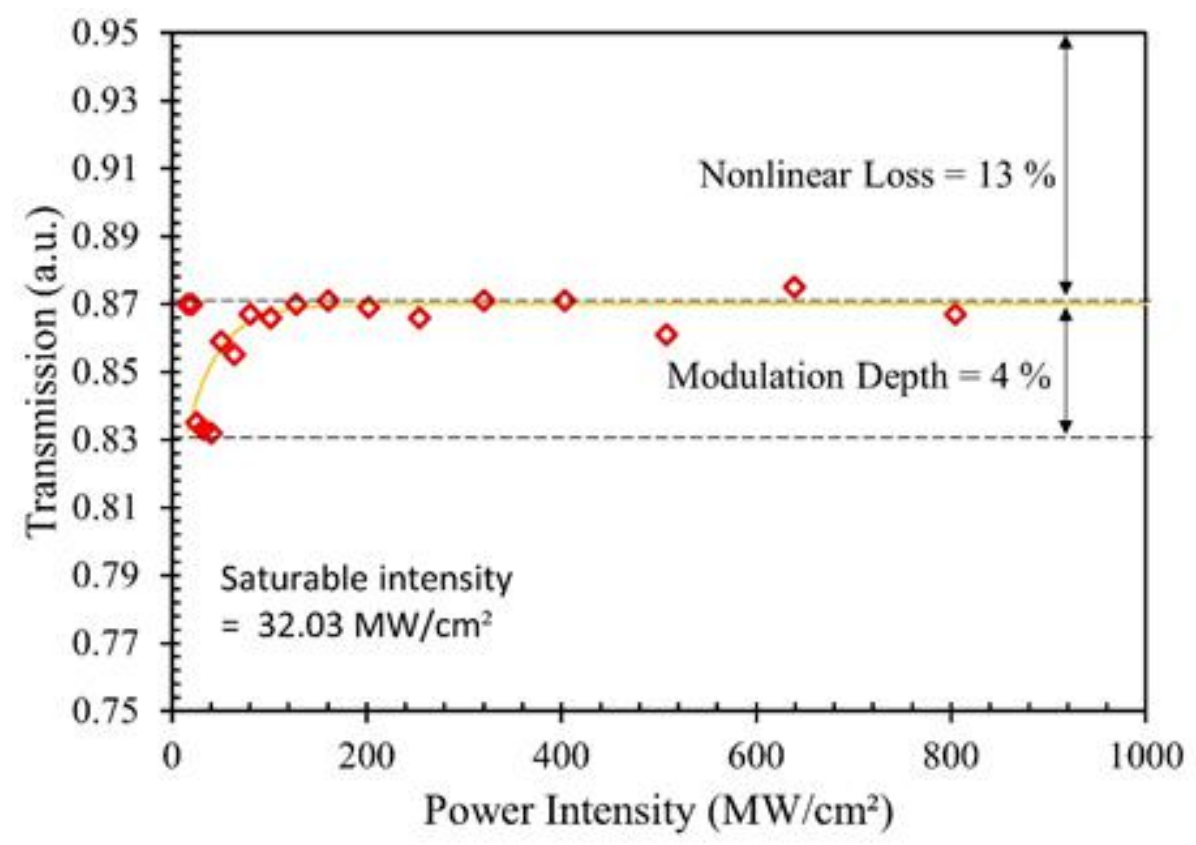

Fig. 1. The nonlinear transmission profile of the lutetium oxide film as SA. 


\section{EXPERIMENTAL SETUP}

The cavity shown in Fig. 2 is composed of a WDM coupler that combines $10 \%$ of the power launched by $980 \mathrm{~nm}$ pump laser diode (LUMICS SN0624200) and 90\% as a feedback signal at $1550 \mathrm{~nm}$ from the cavity. The cavity utilizes $2.4 \mathrm{~m}$ of erbium-doped fiber followed by an isolator to control the signal direction, and then $\mathrm{Lu}_{2} \mathrm{O}_{3}$ connects the isolator to a coupler of 90:10. The polarization independent isolator was used to avoid the light from travelling backwards. The generation of Q-switched was due to the modulation of loss by the $\mathrm{Lu}_{2} \mathrm{O}_{3}$ film incorporated inside the laser cavity. By inserting the SA device inside the laser cavity, the $\mathrm{Lu}_{2} \mathrm{O}_{3} \mathrm{SA}$ absorbed the incoming photon. As the laser pump tuned to a certain pump power value, the electron inside the material can no longer makes a transition between the two energy states, thus, it releases the light in the form of optical pulse (Q-switching generation). As a result of these activities, $\mathrm{Lu}_{2} \mathrm{O}_{3}$ absorbs the emitted signal to generate laser pulses. The Anritsu OSA receives $10 \%$ of the coupler's output to evaluate the signal power and determine the efficiency of the cavity set up. The repetition rate alongside the pulse train are examined by a $6 \mathrm{GHz}$ bandwidth photodetector with a $500 \mathrm{MHz}$ digital oscilloscope with a bandwidth of $4 \mathrm{GHz}$ (GWINSTEK: GDS-3352). The temperature of the laboratory is controlled by keeping the temperature at $20^{\circ} \mathrm{C}$. This is to ensure that the laser diode is in a stable condition. The increase in the surrounding temperature may affect the performance of the generated Q-switched laser. The laser will not work well at high temperature; the heating of laser diode may induce an unstable pulsed laser.

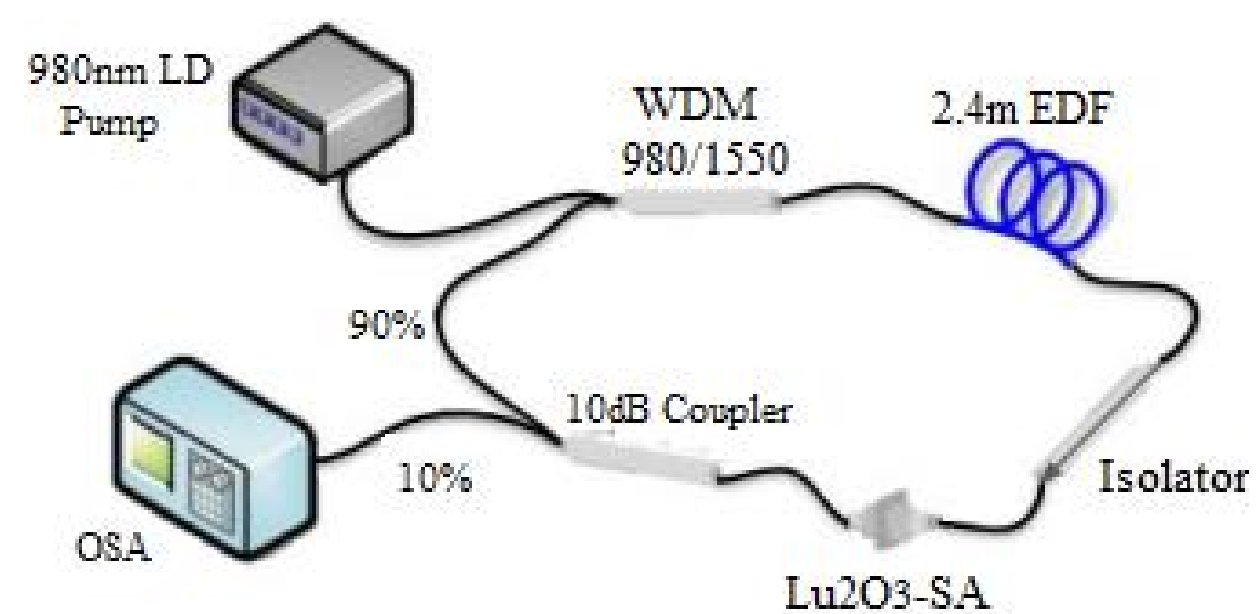

Fig. 2. Fiber laser cavity experimental set-up.

\section{RESULTS AND DISCUSSION}

The laser's output wavelength change is monitored by the OSA device and as it's shown in Fig. 3, the wavelength changed from $1566.7 \mathrm{~nm}$ to $1563.7 \mathrm{~nm}$ at the pumping power of $30.442 \mathrm{~mW}$, when the cavity has the saturable absorber added to it. This change in the wavelength is due to the loss increment in the cavity, hence the laser will emit lower wavelengths, having a greater gain to compensate for the losses. 


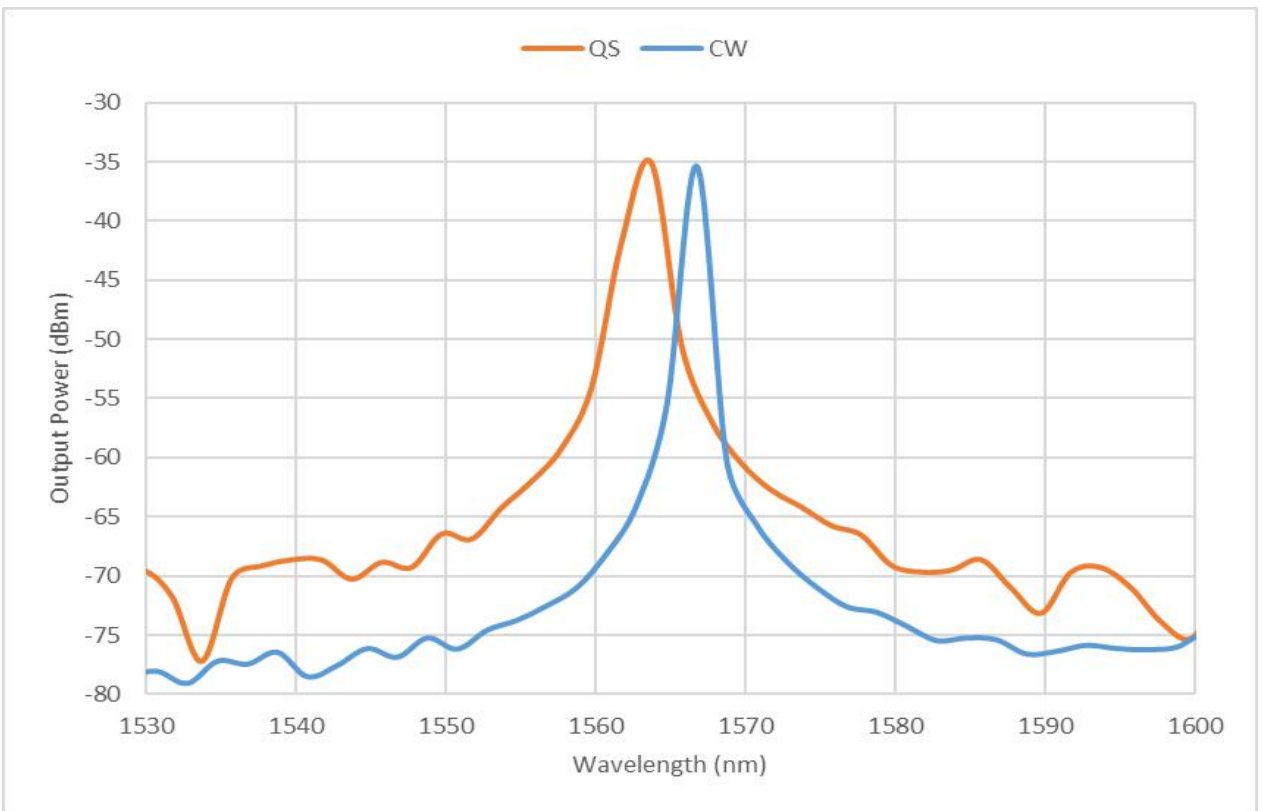

Fig. 3. Continuous wave and Q-switched laser ooutput spectrum

Meanwhile, the repetition frequency increases from $31.25 \mathrm{kHz}$ to $60.2 \mathrm{kHz}$, corresponding to the behavior of a trigger laser pump power which increased from $30.442 \mathrm{~mW}$ and $71.652 \mathrm{~mW}$. On the contrary, the width of the pulse drops from $11.4 \mu$ s to $4.75 \mu$ s as shown in Fig. 4. Clearly from Fig. 5, both laser's output power and pulse energy linearly increased with the pump power. The output power increased from $0.27 \mathrm{~mW}$ to $0.97 \mathrm{~mW}$ while the pulse energy improving from $8.65 \mathrm{~nJ}$ to $16.1 \mathrm{~nJ}$ as the power of the pump was raised from $30.442 \mathrm{~mW}$ and $71.652 \mathrm{~mW}$.

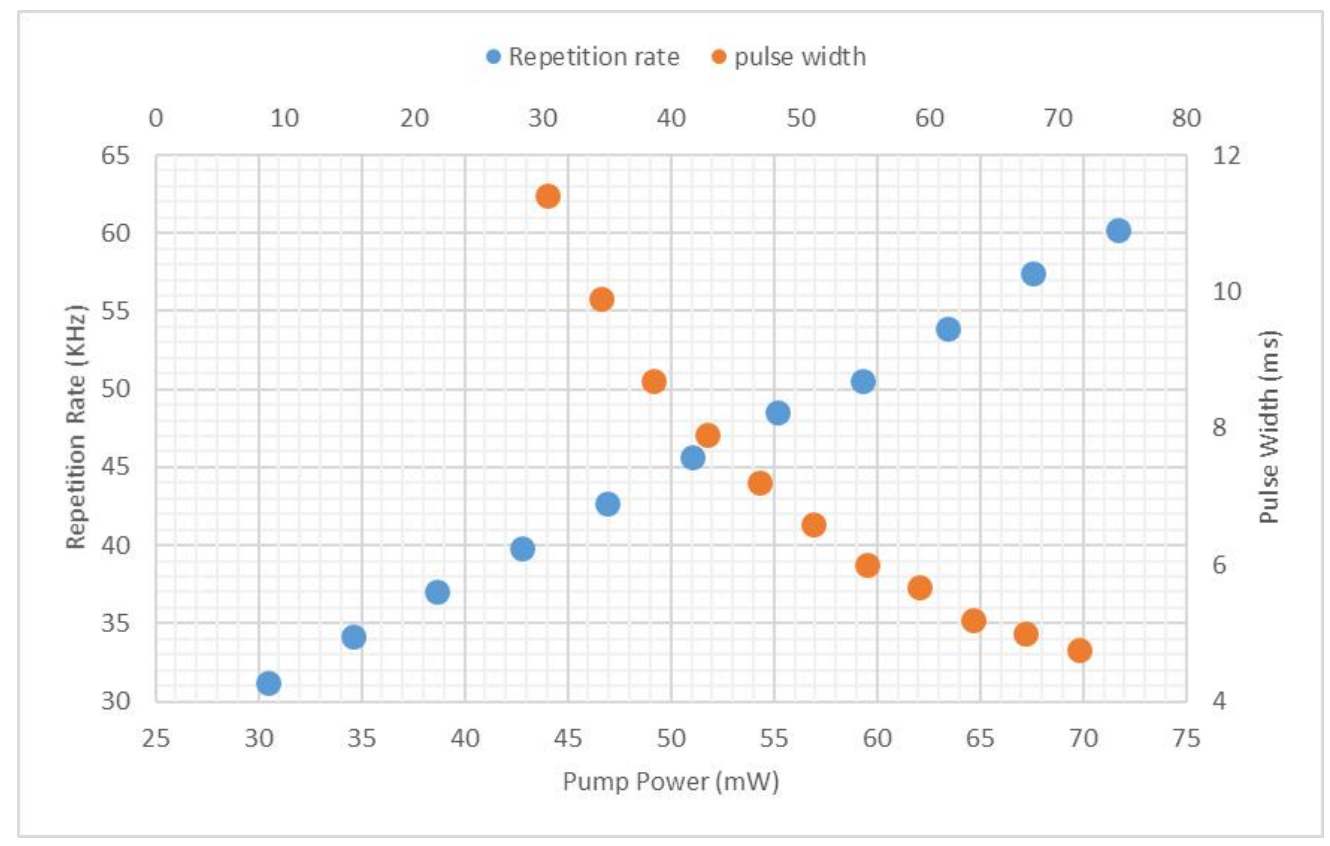

Fig. 4. Repetition rate along with pulse width vs pump power. 


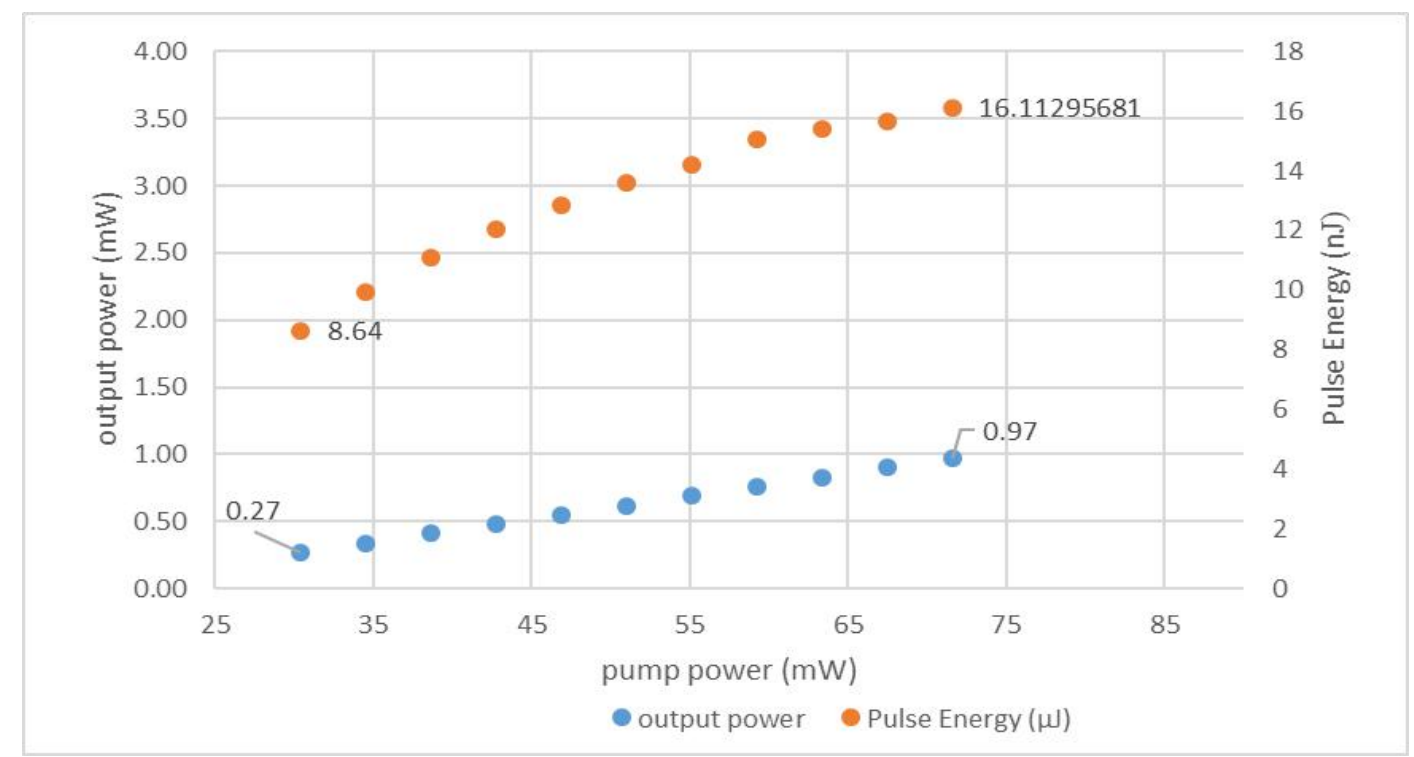

Fig. 5. Output power and pulse energy against pump power.

Fig. 6 shows the typical pulse train for a pump power at $55.17 \mathrm{~mW}$. It shows a peak to peak period of $20 \mu \mathrm{s}$, which corresponds to a repetition rate of $48.6 \mathrm{kHz}$. Fig. 7 shows the RF spectrum when the laser's output power is maximum $(0.97 \mathrm{~mW})$ at a peak for a pump power at $71.652 \mathrm{~mW}$. This provides a low pulse width of $4.75 \mu$ s with a repetition rate equaling to $60.2 \mathrm{kHz}$, indicating that as pumping rises, the pulses frequency increases as shown in Fig. 6. As shown in Fig. 7, a good SNR has been established from the study of the amplitude of the first peak, which allows the determination of the SNR for the frequency that is equal to $55 \mathrm{~dB}$. This confirms the stability of the pulse. The Qswitching generation depends on the saturable absorption mechanism of a nonlinear optical material, which was discussed in our previous work [14].

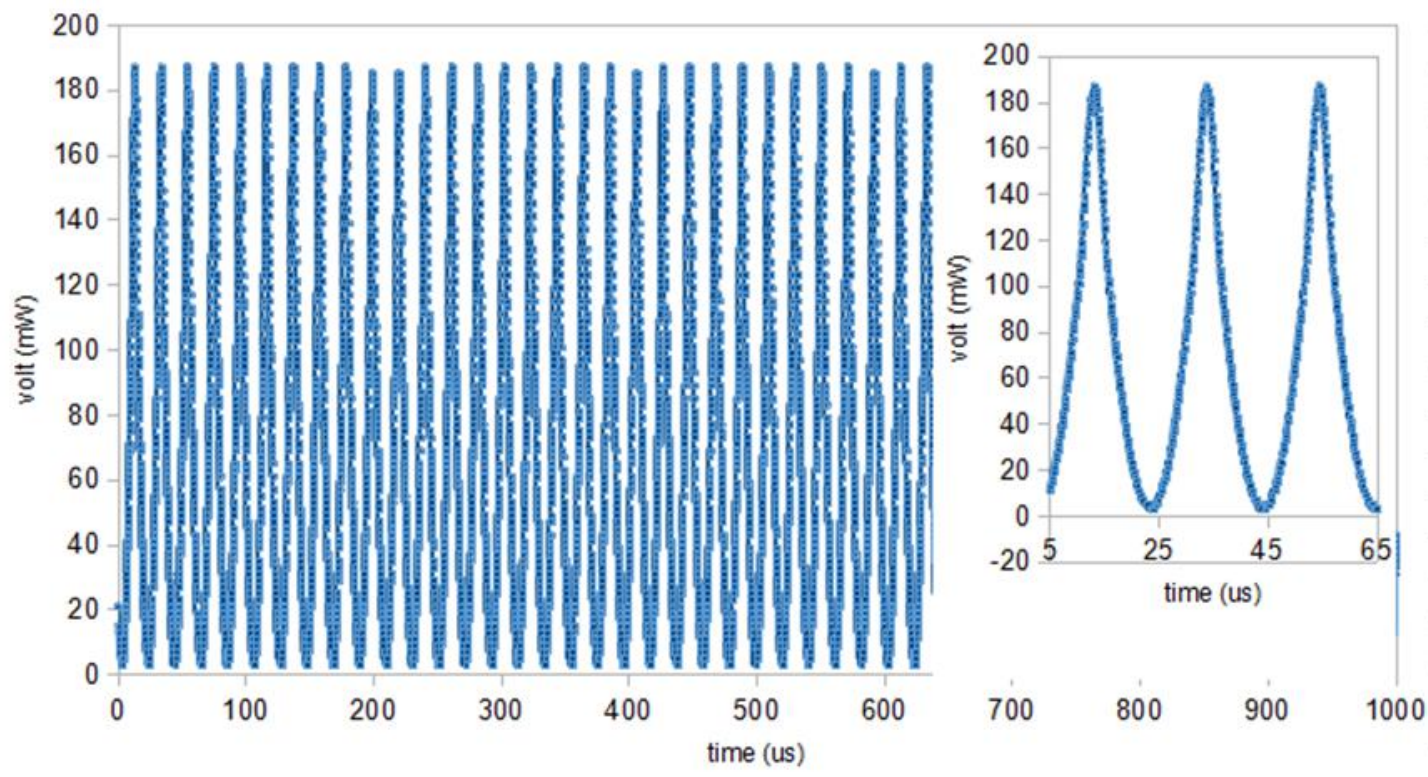

Fig. 6. Oscilloscope trace for the EDFL with $\mathrm{Lu}_{2} \mathrm{O}_{3}$ at $55.17 \mathrm{~mW}$. 


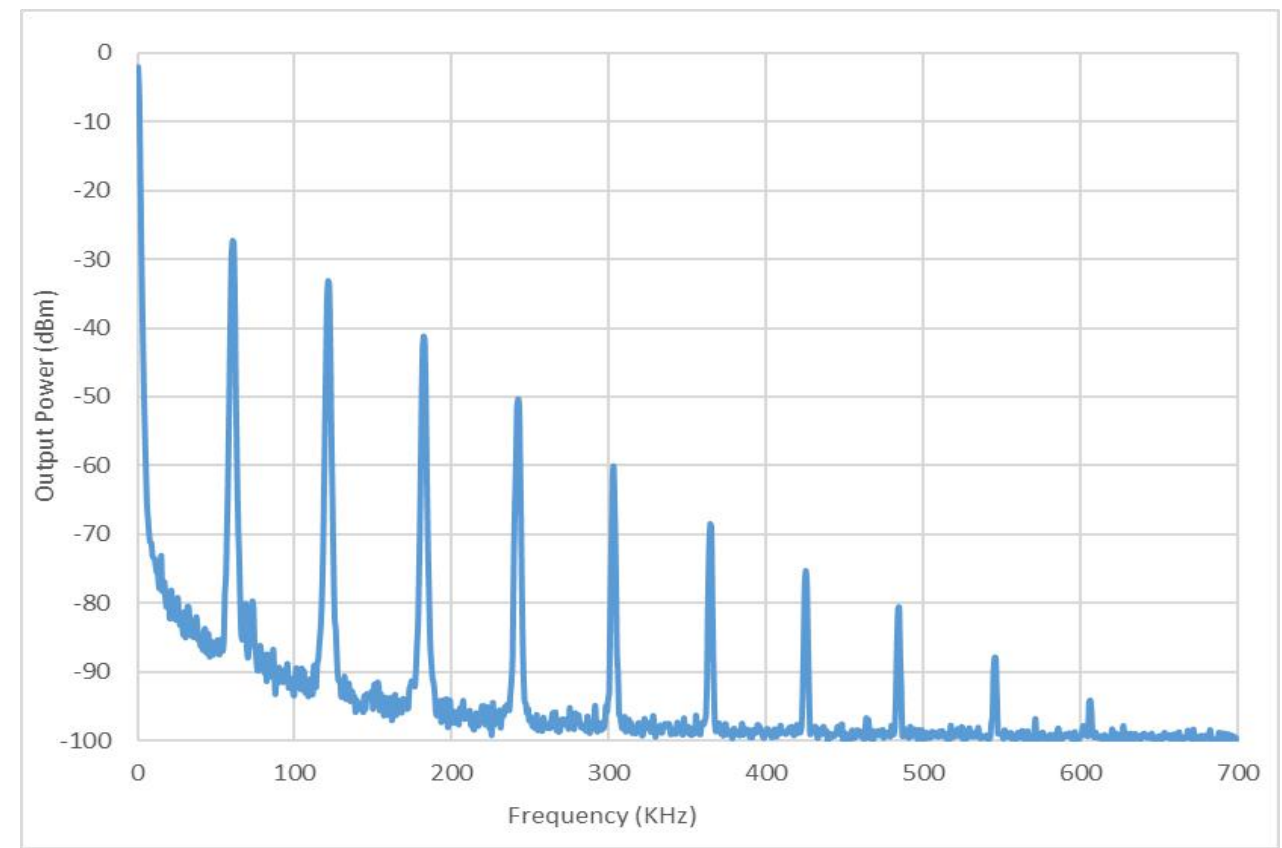

Fig. 7. Radio Frequency Spectrum at maximum output power $0.97 \mathrm{~mW}$

Table I shows a performance comparison of various materials incorporated into the erbium-doped fiber laser cavity as a passive Q-switcher. The developed $\mathrm{Lu}_{2} \mathrm{O}_{3} \mathrm{SA}$ has successfully generated a pulsed laser with a highest signal-to-noise ratio in comparison to other SA materials. The Q-switching threshold is also lower than reduced graphene oxide (r-GO) and black phosphorus (BPs). The generated pulse width and repetition rate was also comparable to other materials. However, the maximum output power produced is low, this is due to the high high intracavity loss inside the developed laser cavity.

TABLE I: THE COMPARISON OF Q-SWITCHING PERFORMANCE BY USING VARIOUS SA MATERIAL IN ERBIUM-DOPED FIBER LASER

\begin{tabular}{|c|c|c|c|c|c|c|}
\hline $\begin{array}{c}\text { SA } \\
\text { materials }\end{array}$ & $\begin{array}{c}\text { Q-switching } \\
\text { Threshold (mW) }\end{array}$ & $\begin{array}{c}\text { Max. Repetition } \\
\text { Rate (kHz) }\end{array}$ & $\begin{array}{l}\text { Min. Pulse } \\
\text { Width }(\mu \mathrm{s})\end{array}$ & $\begin{array}{l}\text { Max Output } \\
\text { Power (mW) }\end{array}$ & SNR & Refs. \\
\hline r-GO & 120 & 116 & 1.85 & 14.6 & - & [15] \\
\hline BPs & 56 & 82.85 & 5.52 & 4.3 & 37 & [16] \\
\hline $\mathrm{MoS}_{2}$ & 15.5 & 27 & 5.4 & 1.7 & 54.4 & [17] \\
\hline $\mathrm{Bi}_{2} \mathrm{Se}_{3}$ & 9.3 & 40.1 & 4.9 & 1.6 & 50 & [18] \\
\hline $\mathrm{Lu}_{2} \mathrm{O}_{3}$ & 30.4 & 60.2 & 4.75 & 0.97 & 55 & $\begin{array}{l}\text { This } \\
\text { work }\end{array}$ \\
\hline
\end{tabular}

\section{CONCLUSION}

A Q-switched pulse laser using EDFL as the gain medium has been experimentally implemented using $\mathrm{Lu}_{2} \mathrm{O}_{3}$ as SA in the cavity and operates at c-band with excellent stability. A stable Q-switched pulse laser is started at the input pump power of $30.442 \mathrm{~mW}$. As the pump power increases from $30.442 \mathrm{~mW}$ to $71.652 \mathrm{~mW}$, the highest energy of the pulse at $16.11 \mathrm{~nJ}$ is measured at an output power 
of $0.97 \mathrm{~mW}$, which confirms the stability of the pulses. As the repetition rate of the Q-switched laser increases from $31.25 \mathrm{kHz}$ to $60.2 \mathrm{kHz}$, the width of the pulse falls from $11.4 \mu \mathrm{s}$ to $4.27 \mu \mathrm{s}$.

\section{ACKNOWLEDGMENT}

The authors would like to thank Photonics Research Laboratory, University of Malaya for their support and assistance with this project.

\section{REFERENCES}

[1] J. Lee, J. Lee, J. Koo and J. H. Lee, "Passively Q-switched erbium-doped fiber laser with graphite saturable absorber based on the pencil-sketching at $1.56 \mu \mathrm{m}$ region," 2016 15th International Conference on Optical Communications and Networks (ICOCN), Hangzhou, pp. 1-2. 2016.

[2] Z. Luo, C. Liu, Y. Huang, D. Wu, J. Wu, H. Xu, Z. Cai, Z. Lin, L. Sun, J. Weng “Topological-Insulator Passively QSwitched Double-Clad Fiber Laser at $2 \mu \mathrm{m}$ Wavelength," IEEE Journal of Selected Topics in Quantum Electronics, vol. 20, no. 5, pp. 1-8. 2014.

[3] M. A. Ismail, F. Ahmad, S. W. Harun, H. Arof and H. Ahmad "A Q-Switched Erbium-Doped Fiber Laser with a Graphene Saturable Absorber," Laser Physics Letters, vol. 10, no. 2, pp. 025102. 2013.

[4] E. Desurvire, J. R. Simpson, and P. C. Becker "High-Gain Erbium-Doped Traveling-Wave Fiber Amplifier," Optics Letters, vol. 12, no. 11, pp. 888. 1987.

[5] M. F. Baharom, M. F. A. Rahman, A. A. Latiff, P. Wang, H. Arof, S. W. Harun "Lutetium Oxide Film as a Passive Saturable Absorber for Generating Q-Switched Fiber Laser at $1570 \mathrm{~nm}$ Wavelength," Optical Fiber Technology, vol. 50, pp. 82-86. 2019.

[6] M. H. M. Ahmed, N. M. Ali, Z. S. Salleh, A. A. Rahman, S. W. Harun, M. Manaf, H. Arof "Q-Switched Erbium Doped Fiber Laser Based on Single and Multiple Walled Carbon Nanotubes Embedded in Polyethylene Oxide Film as Saturable Absorber," Optics \& Laser Technology, vol. 65, pp. 25-28. 2015.

[7] N. N. Razak, A. A. Latiff, Z. Zakaria, S. W. Harun "Q-Switched Erbium-Doped Fiber Laser with a Black Phosphorus Saturable Absorber," Photonics Letters of Poland, vol. 9, no. 2, pp. 72-74. 2017.

[8] L. Zhang, L. Guo, B. Xiong, X. Yan, L. Sun, W. Hou, X. C. Lin, J. M. Li "LD side-pumped high beam quality passive Q-switched and mode-locked Nd:YAG laser based on SESAM," Laser Phys., vol. 20, pp. 1798-1801, 2010.

[9] W. J. Cao, H. Y. Wang, A. P. Luo, Z. C. Luo and W. C. Xu "Graphene-Based, 50 Nm Wide-Band Tunable Passively Q-Switched Fiber Laser,” Laser Physics Letters, vol. 9, no. 1, pp. 54-58. 2011.

[10] R. Krsmanović, Ž. Antić, B. Bártová and M. D. Dramićanin "Characterization of Rare-Earth Doped Lu2O3 Nanopowders Prepared with Polymer Complex Solution Synthesis," Journal of Alloys and Compounds, vol. 505, no. 1, pp. 224-228. 2010.

[11] G. G. Zaimes, B. J. Hubler, S. Wang, and V. Khanna "Environmental Life Cycle Perspective on Rare Earth Oxide Production," ACS Sustainable Chemistry \& Engineering, vol. 3, no. 2, pp. 237-44. 2015.

[12] H. A. Ahsaine, L.Atourki, M. Ezahri, K. Bouabid, A. Ihlal, S. Villain, A. Benlhachemi "Novel Synthesis, Characterization and Optical Properties of $\mathrm{Lu}_{2} \mathrm{O}_{3}$ Deposited by Electrochemical Method," Materials Letters, vol. 160, pp. 415-418. 2015.

[13] R. Lindberg, J. Bogusławski, I. Pasternak, A. Przewłoka, F. Laurell, V. Pasiskevicius, J. Sotor "Mapping ModeLocking Regimes in a Polarization-Maintaining Er-Doped Fiber Laser," IEEE Journal of Selected Topics in Quantum Electronics, vol. 24, no.3, pp. 1-9. 2018.

[14] A. Nady, A. A. Latiff, A. Numan, C. H. R. Ooi, and S. W. Harun "Theoretical and experimental studies on a Qswitching operation in an erbium-doped fiber laser using vanadium oxide as saturable absorber," Laser Physics, vol. 28, no.8, pp. 085106. 2018.

[15] G. Sobon, J. Sotor, J. Jagiello, R. Kozinski, K. Librant, M. Zdrojek, L. Lipinska, K. M. Abramski "Linearly polarized, Q-switched Er-doped fiber laser based on reduced graphene oxide saturable absorber," Applied Physics Letters, vol. 101, no. 24, pp. 4. 2012.

[16] E. I. Ismail, N. A. Kadir, A. A. Latiff, H. Ahmad, S. W. Harun "Black phosphorus crystal as a saturable absorber for both a Q-switched and mode-locked erbium-doped fiber laser," RSC Advances, vol. 6, no. 76, pp. 72692-72697. 2016

[17] Z. Luo, Y. Huang, M. Zhong, Y. Li, J. Wu, B. Xu, H. Xu, Z. Cai, J. Peng, J. Weng "1-, 1.5-, and 2- $\mu$ m Fiber Lasers QSwitched by a Broadband Few-Layer MoS2 Saturable Absorber," Journal of Lightwave Technology, vol. 32, no. 24, pp. 4077-4084. 2014.

[18] L. Sun, Z. Lin, J. Peng, J. Weng, Y. Huang, Z. Luo, "Preparation of Few-Layer Bismuth Selenide by Liquid-PhaseExfoliation and Its Optical Absorption Properties," Scientific Reports, vol. 4, pp. 1-9. 2014. 